\title{
On the Origin, Growth and Application of Ripples
}

\author{
Bert Huis in 't Veld ${ }^{* 1,3}$, Max Groenendijk ${ }^{* 2}$, Hartmut Fischer ${ }^{* 3}$ \\ ${ }^{* 1}$ University of Twente, Faculty of Engineering Technology, Applied Laser Technology, Driener- \\ lolaan 5, Enschede, Post Office Box 217, 7500 AE Enschede, The Netherlands \\ e-mail: a.j.huisintveld@utwente.nl \\ ${ }^{* 2}$ Lightmotif, Drienerlolaan 5, Enschede, Post Office Box 217, 7500 AE Enschede, The Netherlands \\ e-mail:max.groenendijk@lightmotif.nl \\ *3 TNO Science and Industry, Department Materials Technology, De Rondom 1, Eindhoven, Post \\ Office Box 6235, 5600 HE Eindhoven, The Netherlands \\ e-mail hartmut.fischer@tno.nl
}

\begin{abstract}
The origin and growth of a self organizing rippled nanostructure have been studied on slightly etched high temperature steel using $240 \mathrm{fs}$ laser pulses with a wavelength of $800 \mathrm{~nm}$. SEM, AFM and confocal microscopy have been used for analyses. A relatively low energy input results in a regular structure with a wavelength in the range 300 - $500 \mathrm{~nm}$ and with an orientation perpendicular to the polarization direction of the laser light. In twinned areas nearby on the same specimen only "pre ripples" with a much smaller wavelength of about $150 \mathrm{~nm}$ and an amplitude of about 10 $\mathrm{nm}$ are observed. Surprisingly these "pre ripples" have a different orientation. It is proposed that energy absorption within the matrix is strongly influenced by crystal orientation differences and that "pre ripples" can be observed only within a certain energy input range. With increasing energy input regular ripples initiate, here on lines of small intergranular carbides in an area with "pre ripples”. When the energy input is further enhanced the regular rippled pattern is disturbed and finally the ripples transform into disordered structures. Surfaces with a microstructure due to laser ablation and a superimposed rippled texture have been used successfully for creating hydrophobic sur-

DOI: $10.2961 / \mathrm{j} \operatorname{lmn} .2008 .03 .0014$ faces.
\end{abstract}

Keywords: femto, laserpulses, ripples, initiation, nanostructure, twins, steel, SEM, AFM, hydrophobicity

\section{Introduction}

In laser micromachining ripples are frequently observed, the first already in 1965 [1], which is 5 years after the development of the first laser beam. A commonly accepted explanation is that ripples originate from the interference of the incident and reflected or refracted laser light with the scattered or diffracted light near the interface. Scattering of the incident light is supposed to be caused by microscopic roughness of the surface, by defects or by spatial variations in the dielectric constant. The interference between the different waves could lead to an inhomogeneous energy input which, together with positive feedback mechanisms, can cause ripple patterns.

The intensity of the scattered waves depends on the laser parameters and the type of material. If the material possesses an optically active mode near the laser frequency, it is assumed that the scattered light can directly excite a real surface electromagnetic wave (SEW)[2]. Such SEW's or surface polaritons are waves travelling along the interface between two media. If there is no optically active mode near the laser frequency, ripples are supposed to form if one of the diffracted waves propagates close to the surface. It is assumed that the periodicity of ripples depends on the wavelength, the angle of incidence and the polarization of the laser beam [3].

In metals light is almost exclusively absorbed by freefree electron transitions within the conduction band. Within the electron system, the excitation energy is thermalized within typically 10 picoseconds (ps). Thermalization be- tween the electron subsystem and the lattice is much slower, typically longer than $10 \mathrm{ps,} \mathrm{depending} \mathrm{on} \mathrm{the} \mathrm{strength} \mathrm{of}$ the electron-phonon coupling. Thus fs laser excitation generates a hot electron gas and no vapor or plasma plume can develop during the pulse and ablation takes place only after the pulse. The absence of thermal effects makes fs and ps laser pulses very interesting for laser ablation and ripples are attributed to a self organizing nanostructure formation known from ion beam sputtering.

In this study a high temperature steel, alloy $800 \mathrm{H}$ (figure 1), has been used to study the effect of grain orientation and orientation differences due to twinning on the initiation and growth of ripples. To reveal the boundaries between areas with orientation differences, like grains or twins, slightly etched specimen have been used for micromachining experiments.

\section{1 fs laser system}

A titanium sapphire based laser system with a central wavelength of $800 \mathrm{~nm}$ was used for generation of the laser pulses. A seed/oscillator combination (Coherent Vitesse Duo) together with a regenerative amplifier (Coherent RegA9000) delivered pulses at $10 \mathrm{kHz}$ repetition rate with $50 \mathrm{~mW}$ average power. The pulse length, measured by a second order auto correlator, was adjusted to $240 \mathrm{fs}$ for all experiments. A combination of a rotary $\lambda / 2$ wave plate and a beam splitting polarizing cube served as attenuator. The beam is horizontally polarized. Manipulation of the bundle 
over the sample was accomplished by a two mirror galvo scanner system (Scanlab Scangine 14). A $100 \mathrm{~mm}$ f-theta lens (fused silica) focused the beam to a spot size of $25 \mu \mathrm{m}$. Due to the Gaussian energy distribution the effective spot size is smaller, here about $13 \mu \mathrm{m}$. The average fluence was determined by measuring the beam power at the exit of the scanner system with a power meter and dividing it by the repetition rate and the surface area of the laser spot. In all experiments the angle of incidence of laser light was perpendicular to the specimen surface.

\subsection{Alloy $800 \mathrm{H}$}

Alloy $800 \mathrm{H}$ is a highly alloyed (table 1) austenitic steel with primary carbides and the specimen used, contains a high density of intragranular smaller carbides on specific crystal planes. These smaller carbides have grown during industrial service at about $600{ }^{\circ} \mathrm{C}$ during 1 year. These carbides are ideal defects for ripple initiation.

Like many other austenitic steels this alloy contains twins, which are areas with a structure that is mirror symmetrical from the surrounding grain. The specimen has been slightly etched before laser machining during 3 minutes with socalled $\mathrm{V} 2 \mathrm{~A}$ beize, an acid etchant with $\mathrm{HCl}$ and $\mathrm{HNO}_{3}$.

\subsection{SEM, AFM, Confocal light microscopy}

Inspection of surfaces before and after laser treatment has been done by light microscopy followed by Scanning Electron Microscopy (SEM). Measurements of ripple sizes have been done by confocal microscopy and finally with AFM in tapping mode using a super-sharp (radius ca. 5 $\mathrm{nm})$ tip to measure the depth of the smallest ripples.

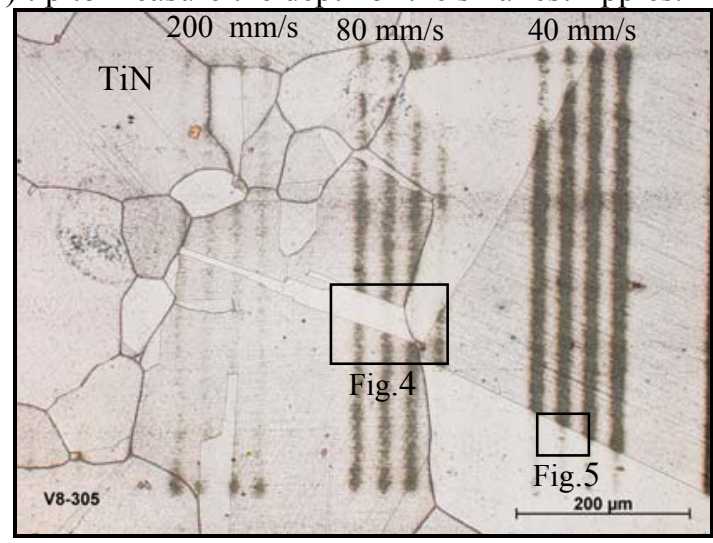

Fig. 1 Etched alloy $800 \mathrm{H}$ with a microstructure dominated by grain boundaries, twins with typical straight boundaries and some yellow coloured primary TiN particles. Three sets of four lines machined by overlap scanning are depicted. The vertical laser micromachining speed was varied from left to right: 200,80 and $40 \mathrm{~mm} / \mathrm{s}$. Other laser conditions: pulse times $240 \mathrm{fs}, \lambda=800 \mathrm{~nm}$, linear polarized, repetition rate $10 \mathrm{kHz}$, fluence $0.4 \mathrm{~J} / \mathrm{cm}^{2}$ and 20 overscans.

Table 1 Chemical composition of X10NiCrAlTi 3220 (alloy $800 \mathrm{H}$ )

\begin{tabular}{|c|c|c|c|c|c|c|c|c|}
\hline $\begin{array}{l}\text { Ele- } \\
\text { ment }\end{array}$ & $\mathrm{Fe}$ & $\mathrm{Ni}$ & $\mathrm{Cr}$ & $\mathrm{Ti}$ & Al & $\mathrm{Mn}$ & $\mathrm{Si}$ & $\mathrm{C}$ \\
\hline Wt $\%$ & bal & $\begin{array}{c}30- \\
34\end{array}$ & $\begin{array}{c}19- \\
23\end{array}$ & $\begin{array}{c}0.15- \\
0.60\end{array}$ & $\begin{array}{c}0.15- \\
0.60\end{array}$ & $\leq 2.0$ & $\leq 1.0$ & $\leq .12$ \\
\hline
\end{tabular}

\section{Rippled surfaces}

Regular ripples on a polished surface of highly alloyed steel (alloy $800 \mathrm{H}$ ) are shown in figure 2. A cube shaped TiN particle is present on the surface. The ripple pattern shows hardly any disturbance due to the presence of the carbide particle.

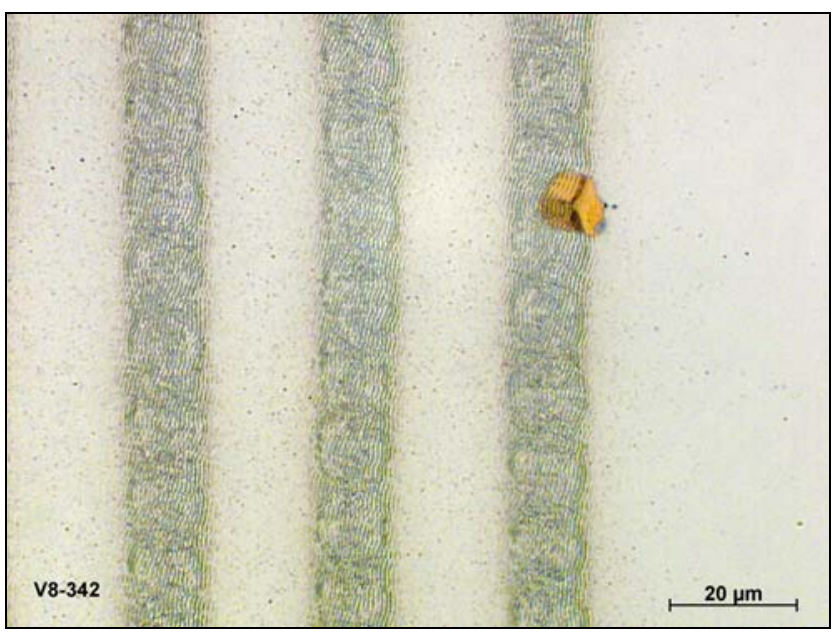

Fig. 2 Light microscopical image of polished surface with three tracks of ripples obtained by scanning a line with $50 \%$ pulse over-

lap. The yellow TiN particle is also rippled. Laser conditions:

fluence $0.15 \mathrm{~J} / \mathrm{cm}^{2}$, scan speed $120 \mathrm{~mm} / \mathrm{s}$ and 10 overscans.

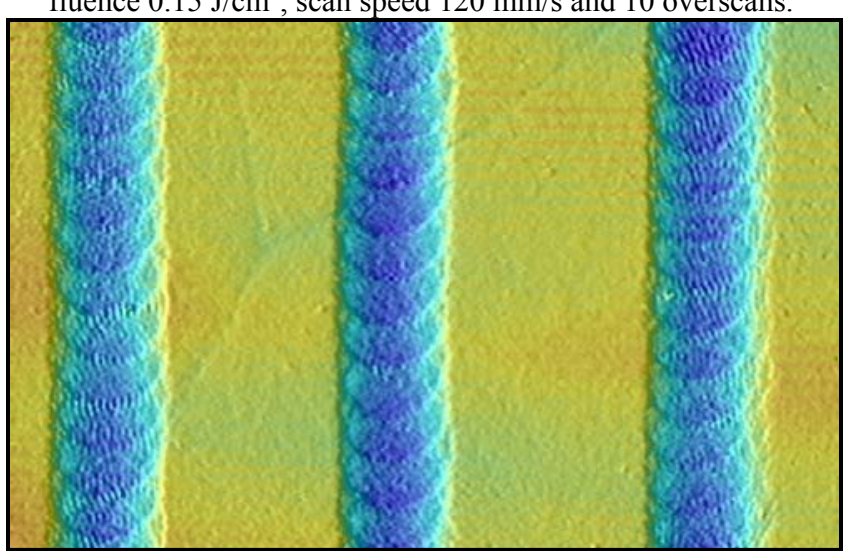

Fig. 3a Confocal microscopical image of rippled tracks on a polished surface obtained with fluence $0.15 \mathrm{~J} / \mathrm{cm}^{2}$ and scan speed 50 $\mathrm{mm} / \mathrm{s}$ with 2 overscans.

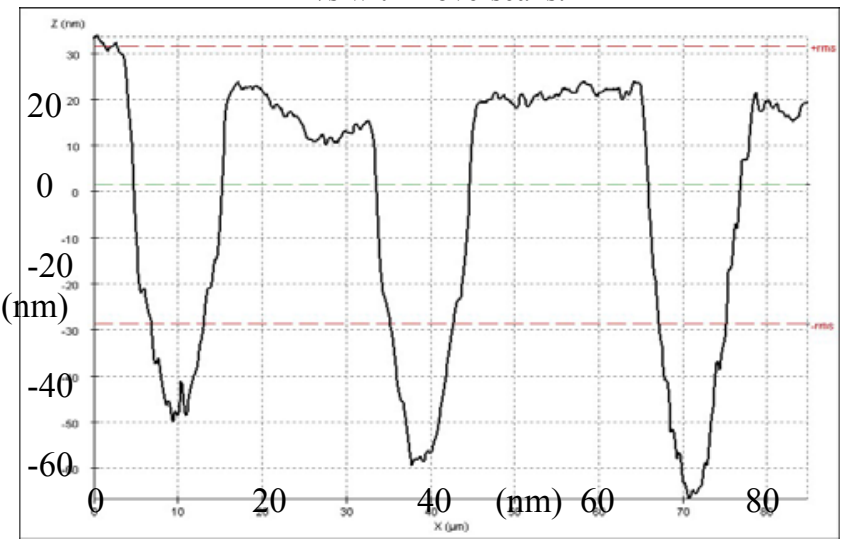

Fig. 3b Depth profile of rippled tracks. In this case the depth is about $80 \mathrm{~nm}$, width is about $13 \mu \mathrm{m}$. Profile has been measured using a confocal light microscope. 
Within the rippled tracks in figure 2 the laser spot size can be recognized. Due to ablation after 10 overscans the strokes have obtained a depth of about $260 \mathrm{~nm}$ as determined by confocal light microscopy. In figure 3 tracks with a width of $13 \mu \mathrm{m}$ and a depth of about $80 \mathrm{~nm}$ are depicted. These figures show the material removal rate, typically 20 $\pm 5 \mathrm{~nm}$ per puls, and the resulting ripples. As can be seen in figure 2 , remarkably enough the ripple pattern is not really disturbed by a TiN particle.

\subsection{The origin of ripples}

Ripple formation comprises the growth of wavy surfaces that can occur on a wide variety of materials. Laser induced ripples show in most cases an orientation perpendicular to the direction of polarization, essential is a driving force along the surface and a certain level of mobility of species on the surface.

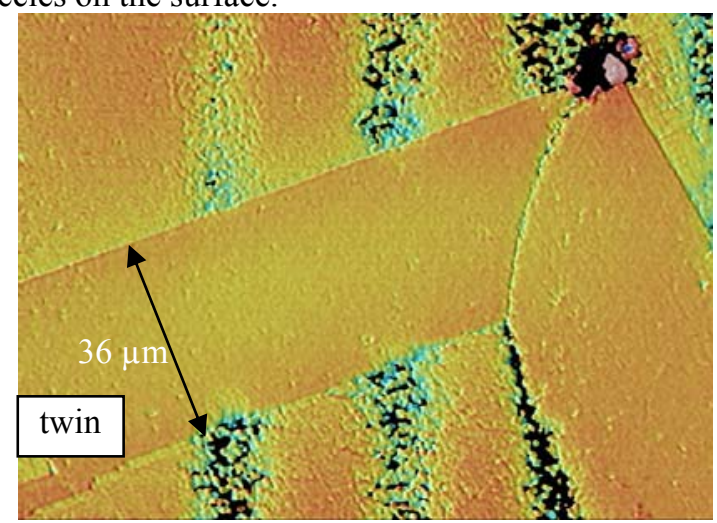

Fig. 4 Confocal image of the central part of Figure 1. Within the twinned area hardly any laser micromachining has occurred.

Conditions used: $80 \mathrm{~mm} / \mathrm{s}$ machining speed, 20 overscans.

Looking at figure 4 the question arises why the laser steel interaction is different in a twinned area with a different crystal orientation. We assume that the etching of the specimen will be different for grains, subgrains or twins with slightly different orientations. The etched surface will not be atomically flat but consist of crystallographic planes with steps on an atomic scale. Due to these small differences the absorption of laser energy in the twinned area is different and apparently reduced. We suggest that the

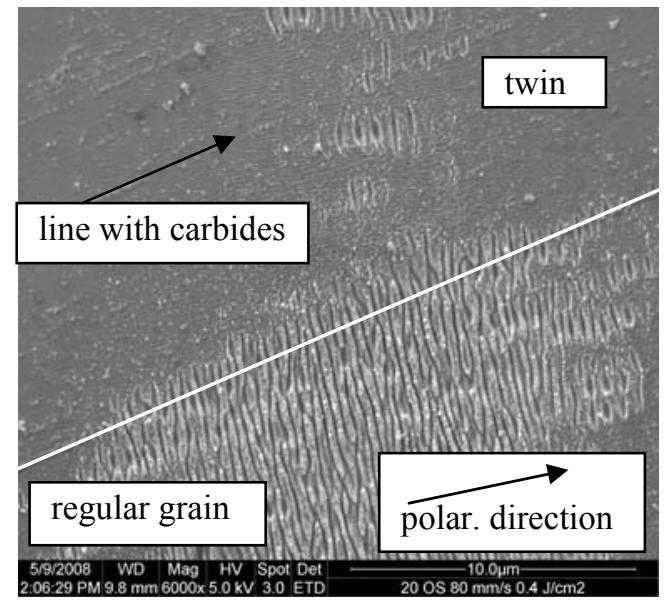

Fig. 5 SEM image of the lower right area of figure 1. Regular ripples perpendicular to the polarization direction are visible. Conditions used $40 \mathrm{~mm} / \mathrm{s}$ machining speed, 20 overscans. energy input remains below the threshold for initiation of ripples.

The effect of a lower machining speed on ablation and ripple formation is visible in figure 1 . A detailed view of the lower right section of figure 1 where a machining speed of $40 \mathrm{~mm} / \mathrm{s}$ is used, is depicted in figure 5 which is a mirror image compared to figure 1. Comparing the areas in figures 4 and 5 , the speed in figure 5 was reduced by a factor of 2 and consequently the energy input was increased by a factor of 2 .

In the upper part of figure 5 a twinned area is depicted. By SEM inspection fine ripples with a different orientation have been found in this area. These fine ripples cover the whole area and we assume they precede the regular larger ripples with an orientation perpendicular to the polarization direction. The fine preceding ripples are called here "preripples". On a few sites within the area of pre-ripples the larger regular ripples have started to initiate on fine secondary carbides which are precipitated in the grains, preferentially on cubic planes appearing as lines in figure 5 .

In the twinned area just outside the area depicted in figure 5 AFM images have been made showing pre-ripples and the onset of larger ripples. The AFM image in figure 6 was made by using a fine AFM tip with a radius of about 5 $\mathrm{nm}$, which made it possible to measure the wavelength and amplitude of the pre-ripples.

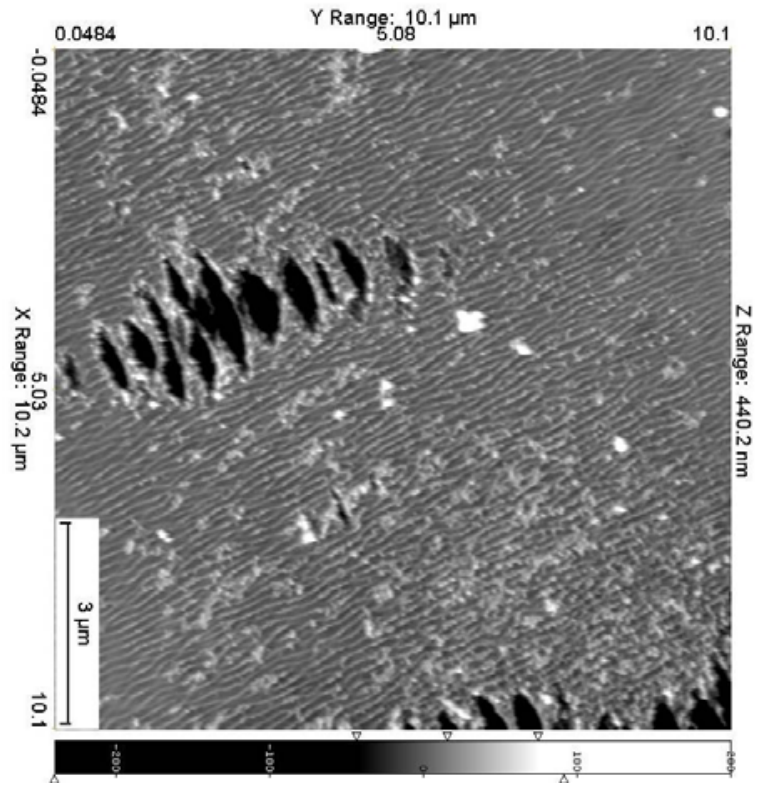

30

20

(nm)

10

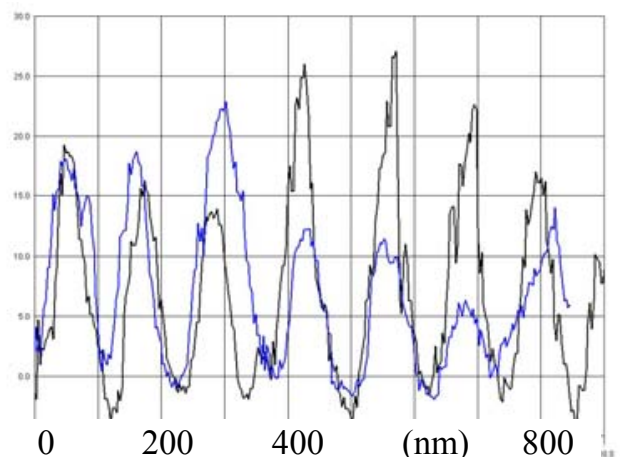

Fig. 6 AFM image and 2 measured profiles of pre ripples. Y axis from $-5 \mathrm{~nm}$ to $30 \mathrm{~nm}$; $\mathrm{x}$ axis from $0 \mathrm{~nm}$ to $900 \mathrm{~nm}$. 
The wavelength of these ripples is about $150 \mathrm{~nm}$ and the amplitude is in the range $8-13 \mathrm{~nm}$.

After the first observation of pre-ripples the test specimen have been inspected again and indeed the finer preripples appeared to be present on other sites different from the originally inspected. All pre-ripples found had the same orientation, therefore, the orientation is not dependent on crystal orientation but influenced by laser micro machining conditions. Pre-rippled areas are found between areas with regular ripples and have been obtained after micromachining with a very low energy input. Obviously for the formation of pre-ripples the energy level should be above the threshold for pre-ripples and below the threshold for initiation of regular ripples. Increasing of the energy level leads to a surface completely covered with regular ripples. Apparently the crystal orientation has a strong effect on the energy absorption; this can be seen in figure 1. Some orientations are favorable for incoupling of energy, however small orientation differences of grains and subgrains, and differences due to twinning can have large effects. Similar effects are observable using ion beam machining, where rippled surfaces have also been observed. The formation and orientation of the ripples seem to be very dependant on ion-beam incidence angles and crystallographic orientations of the specimen surface $[4,5]$. For the laser experiments reported here the crystallographic orientation is most likely influencing the amount of laser energy absorption. However, other crystallographic effects cannot be excluded completely, e.g. surface transport and preferential mobility along crystallographic directions could play a role as well.

\subsection{The growth of ripples}

After initiation ripples will start to grow both in wavelength and in amplitude. An exponentially fast growth at the beginning followed by saturation to a slower growth is predicted. The morphological evolution of surfaces during ion sputtering has been compared wit aeolian ripple formation in sandy deserts. Although the phenomena are physically different they must obey similar geometrical constraints. The numerical solutions show e.g. surface ripple rotation by varying the incident angle [6].

In figure 7 different ripple wavelengths can be observed, larger in the center and smaller at the edge. The larger wavelength of about $400 \mathrm{~nm}$ is due to an intensity effect [7] or a temperature effect as has been found in ion beam sputtering experiments [8]. The smaller wavelength at the edge is about $250 \mathrm{~nm}$. The periodic ripple patterns obtained by mild processing conditions, transform while decreasing the machining speed into more chaotic structures, as depicted in figure 8 [9]. These results have been obtained on stainless steel 304 . In figure 8 the different steps in the transformation are visible. Within the rippled patterns a second orientation is already visible in figure $8 \mathrm{a}$, and this orientation becomes stronger, see $8 \mathrm{c}$ and $8 \mathrm{~d}$. Finally this new orientation becomes dominant and disturbs the regularity.

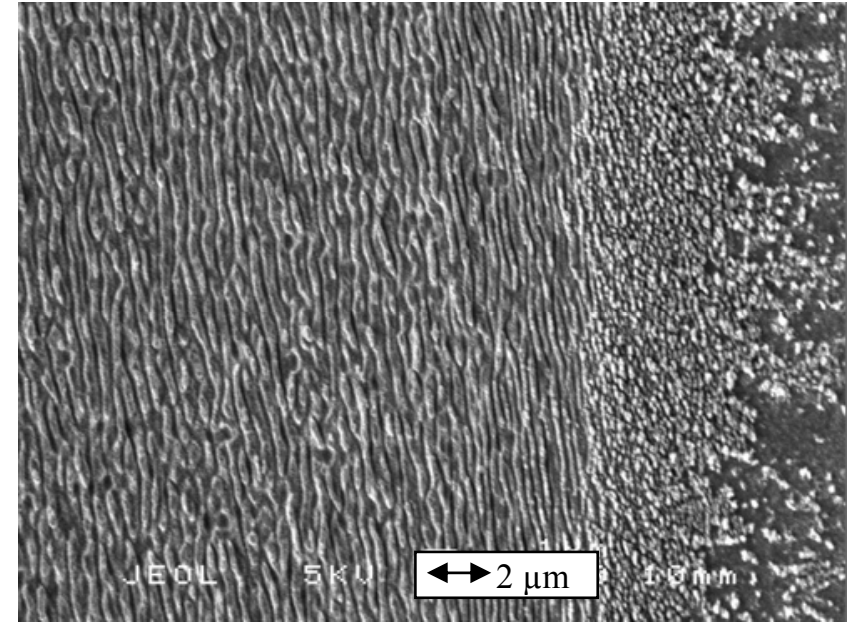

Fig. 7 SEM image of regular ripples with some debris on the right hand side of the rippled area. In the centre of the stroke the wavelength is larger than at the edge.

By comparing the figures 8 and 5 , it can be noticed that the new orientation disturbing the regular ripple pattern is close to the orientation of pre-ripples.
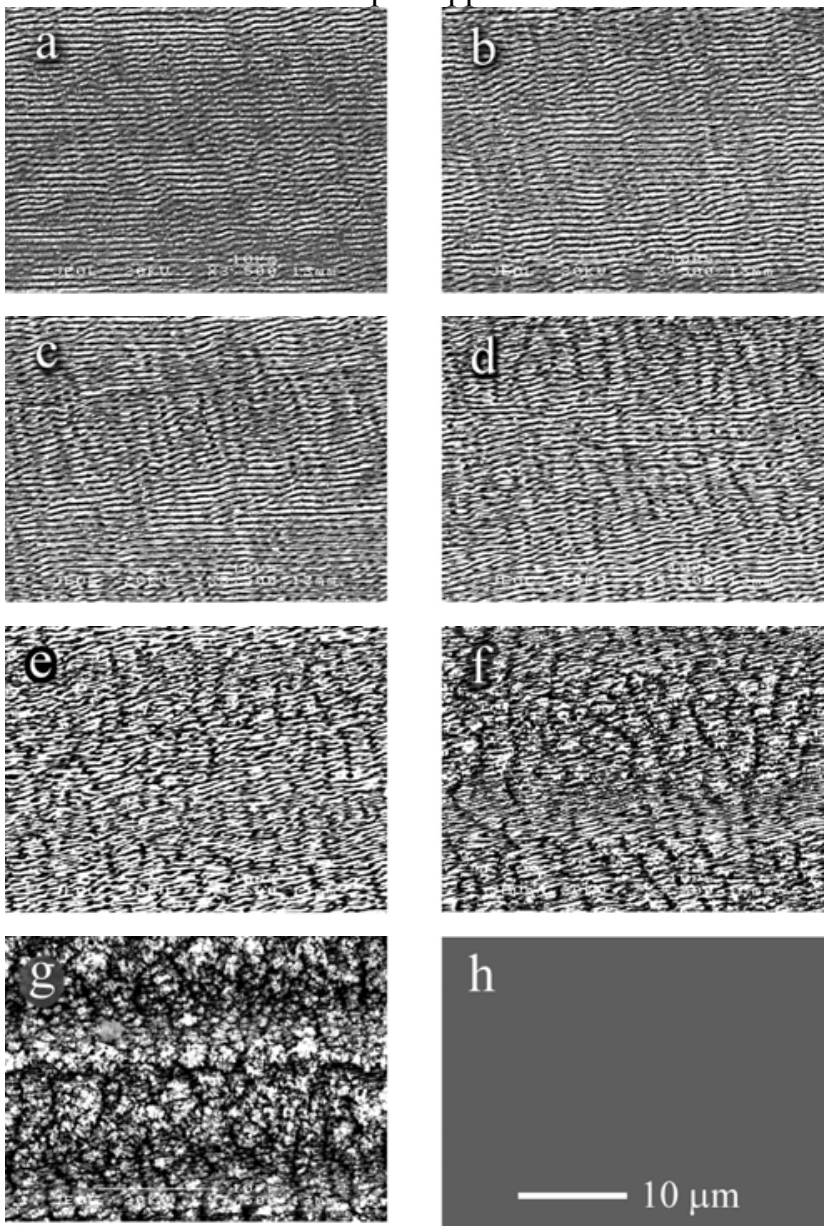

Fig. 8 SEM images of surface structures in stainless steel obtained by femto second laser pulses. The periodic ripple patterns obtained by mild processing conditions (a-b), transform into more chaotic structures $(\mathrm{c}-\mathrm{g})$ as the machining speed decreases.

a) $2000 \mathrm{~mm} / \mathrm{s}$, b) $1000 \mathrm{~mm} / \mathrm{s}, \mathrm{c}) 500 \mathrm{~mm} / \mathrm{s}, \mathrm{d}) 250 \mathrm{~mm} / \mathrm{s}$, e) 100 $\mathrm{mm} / \mathrm{s}, \mathrm{f}$ ) $50 \mathrm{~mm} / \mathrm{s}, \mathrm{g}$ ) $16 \mathrm{~mm} / \mathrm{s}$ (Fluence $1.1 \mathrm{~J} / \mathrm{cm}^{2}$ ) .

Further experiments and analysis are required to verify the hypothesis that the orientation of pre ripples returns in 
the regular ripples and contributes to the disturbance. If this hypothesis can be verified it could imply that the regular ripples form an intermediate surface state.

\section{The application of ripples}

By surface ablation with ultra short laser pulses a well controlled micro textured surface can be obtained. This microstructure is primarily controlled by the effective laser spot size. By superimposing the self organizing rippled nanostructure to the microstructure a double patterned surface can be made. Such a laser micro-nano textured surface is depicted in figure 9. It has been shown that these surfaces have super-hydrophobic properties [10]. In fact by controlling the surface texture, both the microstructure and the superimposed nanostructure, it is possible to control the degree of hydrophobicity which can be quantified by contact angle measurement. Furthermore, by producing a master surface, reproduction on larger scale for instance in polymer surfaces by injection molding or other replication technologies is becoming a technologically feasible process. Figure 10 depicts the effect of the surface texture on the contact angle of a water drop. Here, two drops are placed on an injection molded sample, where the left area is textured and the right area is flat.
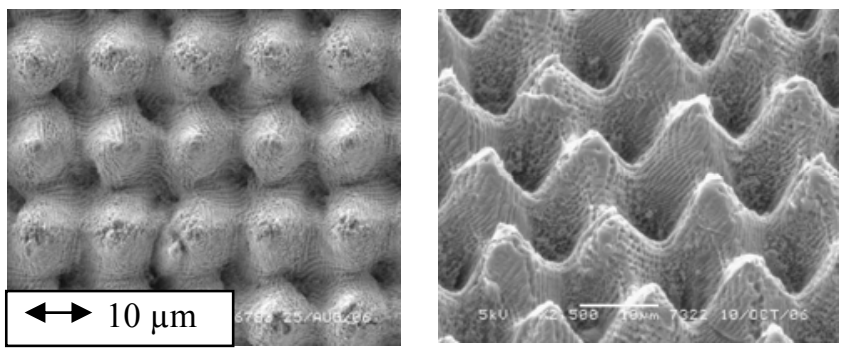

Fig. 9 Left: SEM image of micro-nano textured surface structure in stainless steel obtained by femto second laser pulses. Right: Injection molded replica of left surface structure. These surfaces have super hydrophobic properties.

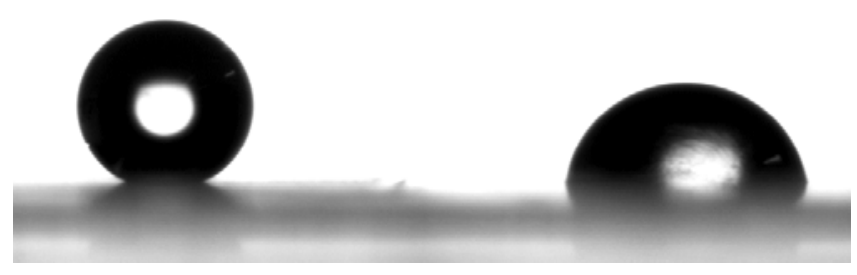

Fig. 10 Photograph of two water drops on a polymer sample. Left on micro/nano structured area, right on flat substrate.

\section{Discussion and Conclusions}

Evidence has been presented that laser micromachining of steel surfaces with 240 fs laser pulses generates two different ripple orientations. For low energy input pre-ripples are observed with an amplitude of about $10 \mathrm{~nm}$ and a wavelength of about $150 \mathrm{~nm}$. These pre-ripples have an orientation far from perpendicular to the polarization direction of laser light. It is proposed that a certain threshold in energy in- coupling should be passed to transform these pre-ripples into regular ripples with an orientation perpen- dicular to the polarization of laser light. The transformation process is visualized in figures 5 and 6 . The wavelength of the larger regular ripples in figures 5 and 7 is in the range of $0.2 \mu \mathrm{m}-0.5 \mu \mathrm{m}$ and the amplitudes are in the range of $50 \mathrm{~nm}-100 \mathrm{~nm}$. Regular ripples initiate on lines of small carbides present in the alloy used for these experiments.

Pre ripples (figure 6), varying ripple spacing (figure 7) and machine speed dependence (figure 8) are not consistent with the model of an interference modulated energy input transforming directly into the observed surface modulation. The model of self organizing structure formation can explain varying spacings [6]. However the origin of pre ripples remains unexplained. Especially the different orientation of pre ripples and the transition towards regular ripples requires further attention.

All ripples observed have wavelengths (much) smaller than the laser light $(800 \mathrm{~nm})$ applied. The interaction of the incident laser light with defects leads to the initiation of pre ripples. Once a surface is covered with pre-ripples, the interaction with the incident laser light is expected to change.

It has been noticed that within the regular rippled surfaces, of previously micro machined ordinary stainless steel, a second orientation is present (figure 8). This orientation becomes stronger when the energy input is enhanced and it leads finally to complete disturbance of regularity. The hypothesis that the orientation of pre ripples returns, leading to disorder has to be verified.

The challenge is to develop a well controlled laser surface texturing process and to model the hydrophobicity of a micro-nano textured surface. The potential of laser micromachining for hydrophobicity is illustrated in figure 10 . When process and model are available laser textured surfaces with unique and predictable properties can be produced.

\section{References}

[1] M. Birnbaum, Journal of Applied Physics, 36, (1965) 3688.

[2] M.N. Libenson, V.S. Makin, V.A. Shiryaev, M.J. Soieau, Proceedings of SPIE, The International Society for Optical Engineering, Vol 2428, (1995) 676-681.

[3] D. Bauerle: "Laser Processing and Chemistry" $3^{\text {rd }}$ ed., 2000.

[4] H.X. Qian, W. Zhou, Y.Q. Fu, B.K.A. Ngoi, G.C. Lim, Applied Surface Science 240 (2005) 140-145

[5] U. Wendt, G.Nolze, H.Heyse, Microsc Microanal 12 (Supp2), (2006) 1302

[6] T. Aste and U. Valbusa, New Journal of Physics 7 (2005) 122

[7] J. Reif, F.Costache, M. Bestehorn, Recent advances in Laser Processing of Materials, pp 275 - 290, Elsevier 2006

[8] M.M. Ovsyanko, Thesis University of Twente, Ion sculpting of $\mathrm{Cu}(001), 2006$

[9] M. Groenendijk and J. Meijer, Journal of Laser Applications, Vol 18, 3 (2006) 227

[10] M. Groenendijk, Laser Technik Journal, Volume 5, Issue 3 (2008) page $44-47$.

(Received: July 1, 2008, Accepted: December 9, 2008) 